\title{
Persistent Spin Dynamics Intrinsic to Amplitude-Modulated Long-Range Magnetic Order
}

\author{
M. Pregelj, ${ }^{1,2}$ A. Zorko, ${ }^{1,3}$ O. Zaharko, ${ }^{2}$ D. Arčon, ${ }^{1,4}$ M. Komelj, ${ }^{1}$ A. D. Hillier, ${ }^{5}$ and H. Berger ${ }^{6}$ \\ ${ }^{1}$ Jožef Stefan Institute, Jamova cesta 39, 1000 Ljubljana, Slovenia \\ ${ }^{2}$ Laboratory for Neutron Scattering, PSI, CH-5232 Villigen, Switzerland \\ ${ }^{3}$ EN-FIST Centre of Excellence, Dunajska 156, SI-1000 Ljubljana, Slovenia \\ ${ }^{4}$ Faculty of Mathematics and Physics, University of Ljubljana, Jadranska cesta 19, SI-1000 Ljubljana, Slovenia \\ ${ }^{5}$ ISIS Facility, Rutherford Appleton Laboratory, Chilton, Didcot, Oxon OX11 OQX, United Kingdom \\ ${ }^{6}$ École Polytechnique Fédérale de Lausanne, Lausanne, Switzerland
}

(Received 21 August 2012; published 27 November 2012)

\begin{abstract}
An incommensurate elliptical helical magnetic structure in the frustrated coupled-spin-chain system $\mathrm{FeTe}_{2} \mathrm{O}_{5} \mathrm{Br}$ is surprisingly found to persist down to 53(3) $\mathrm{mK}\left(T / T_{N} \sim 1 / 200\right)$, according to neutron scattering and muon spin relaxation. In this state, finite spin fluctuations at $T \rightarrow 0$ are evidenced by muon depolarization, which is in agreement with specific-heat data indicating the presence of both gapless and gapped excitations. We thus show that the amplitude-modulated magnetic order intrinsically accommodates contradictory persistent spin dynamics and long-range order and can serve as a model structure to investigate their coexistence.
\end{abstract}

DOI: 10.1103/PhysRevLett.109.227202

PACS numbers: 75.25. $-\mathrm{j}, 75.30 . \mathrm{Fv}, 75.40 . \mathrm{Gb}, 76.75 .+\mathrm{i}$

Geometrical frustration is a common precursor for exotic magnetic ground states-from long-range ordered (LRO) incommensurate (IC) spiral states to highly disordered frozen spin-glass states [1]. It can even lead to spin liquids, where spin fluctuations may endure down to zero temperature-persistent spin dynamics (PSD) [2] is present. Such behavior is typically found in highly frustrated pyrochlore and kagome spin systems with macroscopically degenerate ground states [3]. Since spin fluctuations hinder the onset of extended static correlations, PSD and LRO are generally considered mutually exclusive. Remarkably, their coexistence in the same phase has been reported in several frustrated magnetic systems [4-11] but still lacks a suitable explanation.

To explore this phenomenon, we focus on the $\mathrm{FeTe}_{2} \mathrm{O}_{5} \mathrm{Br}$ multiferroic, in which the magnetic exchange network consists of alternating $\mathrm{Fe}^{3+}(S=5 / 2)$ spin chains coupled by weaker frustrated interactions within the $b c$ layers [12] (Fig. 1). The magnetic order at $5 \mathrm{~K}$, i.e., well below the Néel temperature $T_{N}=10.5 \mathrm{~K}$, was described as an IC collinear amplitude-modulated (AMOD) structure, with magnetic vector $\mathbf{q}=\left(\frac{1}{2} 0.4630\right)$ [13]. In such a state, only part of the total $\mathrm{Fe}$ magnetic moment at each site contributes to LRO, while its counterpart (at each site) is disordered and may fluctuate. Generally, on cooling the ordered component fully develops, which manifests either as a "squaring" of the AMOD structure [14] or as a consecutive transition, where a perpendicular ordered component develops leading to a circular helix [15]. Since $\mathrm{FeTe}_{2} \mathrm{O}_{5} \mathrm{Br}$ exhibits no subsequent transition down to $300 \mathrm{mK}$ [16], it is possible that PSD and LRO coexist.

In this Letter, we report on a combined study of spherical neutron polarimetry (SNP) and neutron diffraction, which reveals that the IC AMOD magnetic structure persists to the lowest accessible temperatures $\left(T / T_{N} \sim\right.$ $1 / 175)$. This is consistent with muon spin relaxation ( $\mu \mathrm{SR}$ ) measurements at $T / T_{N} \sim 1 / 200$, which in addition to static LRO signify the presence of PSD. The coexistence of LRO and PSD is supported by specific-heat data, indicating gapped as well as gapless magnetic excitations. Our study suggests that this is intrinsic to AMOD magnetic

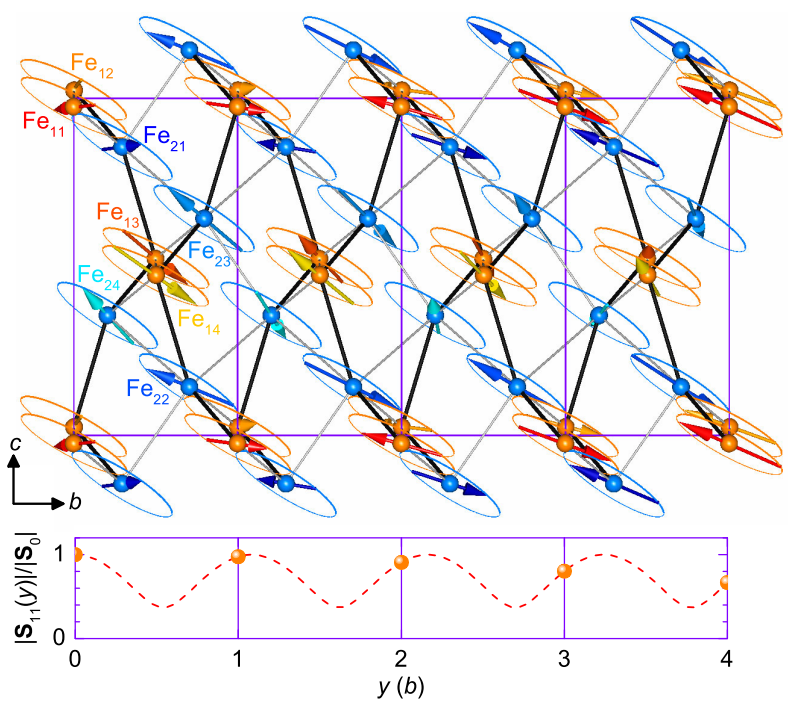

FIG. 1 (color online). Top: Magnetic structure in $\mathrm{FeTe}_{2} \mathrm{O}_{5} \mathrm{Br}$ at $2 \mathrm{~K}$. Light (orange) and dark (blue) spheres are $\mathrm{Fe}_{1}$ and $\mathrm{Fe}_{2}$ magnetic ions, respectively. The shades (colors) of the magnetic moments (arrows) are different for the eight magnetically inequivalent sites $\mathrm{Fe}_{m n}$. The amplitude of the moment changes with its orientation within the ellipsoidal envelope. Black and gray lines denote the dominant intrachain and the weaker interchain exchange interactions, respectively. Bottom: IC amplitude modulation of the $\mathrm{Fe}_{11}$ magnetic moment along the $b$ axis. 
structures. It offers a well-defined framework and a coherent explanation for the coexistence of LRO and PSD that has been missing for the known cases [11].

We begin by a reinvestigation of the $\mathrm{FeTe}_{2} \mathrm{O}_{5} \mathrm{Br}$ magnetic ground state, combining single-crystal neutron diffraction and SNP, which was proven before to be invaluable for determination of complex magnetic structures [17]. The experiments were performed at 5 and $2 \mathrm{~K}$, respectively, on high-quality single crystals [13] with an average size of $15 \times 8 \times 2 \mathrm{~mm}^{3}$ at the Swiss Neutron Spallation Source, Paul Scherrer Institute (PSI), Switzerland. For SNP the MuPAD device on the triple axis spectrometer TASP $(\lambda=3.2 \AA)$ was used, while the diffraction experiment employed the single-crystal diffractometer TriCS $(\lambda=2.317 \AA)$. Polarization matrices were measured for three different crystal orientations [18], accessing for the first time [13,19] also $h k l \pm \mathbf{q}(l \neq 0)$ magnetic reflections.

The general magnetic structure model, in which the magnetic order breaks all crystallographic symmetry operations $[13,19]$, dictates the magnetic moment at a particular Fe site to follow an elliptical helix with the pitch along the magnetic $\mathbf{q}$ vector:

$$
\mathbf{S}_{m n}\left(\mathbf{r}_{i}\right)=\mathbf{S}_{0 m n}^{\mathrm{Re}} \cos \left(\mathbf{q} \cdot \mathbf{r}_{i}-\psi_{m n}\right)+\mathbf{S}_{0 m n}^{\mathrm{Im}} \sin \left(\mathbf{q} \cdot \mathbf{r}_{i}-\psi_{m n}\right) .
$$

Here, vector $\mathbf{r}_{i}$ defines the origin of the $i$ th cell, $m=1,2$ identifies the crystallographically inequivalent $\mathrm{Fe}$ sites, and $n=1-4$ denotes the four $\mathrm{Fe}$ positions within the crystallographic unit cell (see the caption of Table I). The complex vector $\mathbf{S}_{0 m n}$ is determined by its real and imaginary components $\mathbf{S}_{0 m n}^{\mathrm{Re}}$ and $\mathbf{S}_{0 m n}^{\mathrm{Im}}$, respectively, defining the amplitude and the orientation of the magnetic moments, while $\psi_{m n}$ denotes a phase shift. We assume the same moment $\mathbf{S}_{0 m n} \equiv \mathbf{S}_{0 m}$ for all crystallographically equivalent $\mathrm{Fe}$ sites. The reliability of the refined magnetic structure is ensured by simultaneous refinement of all SNP and integrated magnetic-peak intensities data [18]. The best solution (Fig. 1 and Table I) with the dominant component pointing $\sim 45^{\circ}$ away from $a$ towards the $-b$ axis agrees

TABLE I. Parameters of the best magnetic structure model at $2 \mathrm{~K}$ for two independent magnetic atoms $\left(\mathrm{Fe}_{1}\right.$ and $\left.\mathrm{Fe}_{2}\right)$, and eight magnetic phases $\psi_{m n}$, i.e., one for each of the magnetic $\mathrm{Fe}_{m n}$ atoms in the unit cell $(m=1,2, n=1-4)$. The sites $\mathrm{Fe}_{12}-\mathrm{Fe}_{14}$ are obtained from $\mathrm{Fe}_{11}[0.1184(6),-0.001(1), 0.9734(7)]$ and $\mathrm{Fe}_{22}-\mathrm{Fe}_{24}$ from $\mathrm{Fe}_{21}$ [0.9377(6), 0.2953(1), 0.8562(6)] by symmetry elements $i, 2_{1 y}$, and $2_{1 y} i$, respectively. The orientation of the moments is given in the $a^{*} b c$ coordinate system.

\begin{tabular}{lrrrrrrr}
\hline \hline$s=\mathrm{Re}, \mathrm{Im}$ & \multicolumn{1}{c}{$\mathrm{Fe}_{1}^{\mathrm{Re}}$} & \multicolumn{1}{c}{$\mathrm{Fe}_{1}^{\mathrm{Im}}$} & \multicolumn{1}{c}{$\mathrm{Fe}_{2}^{\mathrm{Re}}$} & \multicolumn{1}{c}{$\mathrm{Fe}_{2}^{\mathrm{Im}}$} & $n$ & $\psi_{1 n}$ & $\psi_{2 n}$ \\
\hline$S_{0 x}^{s} /\left|S_{0}^{s}\right|$ & 0.70 & 0.71 & 0.64 & 0.76 & 1 & 0.00 & 0.00 \\
$S_{0 y}^{s} /\left|S_{0}^{s}\right|$ & 0.70 & -0.67 & 0.76 & -0.61 & 2 & 0.04 & 0.93 \\
$S_{0 z}^{s} /\left|S_{0}^{s}\right|$ & -0.14 & 0.20 & -0.10 & 0.21 & 3 & 0.17 & 0.20 \\
$\left|\mathbf{S}_{0 m}^{s} / /\right| \mathbf{S}_{0} \mid$ & 0.39 & 0.96 & 0.35 & 1.00 & 4 & 0.21 & 0.15 \\
\hline \hline
\end{tabular}

with the previously proposed simplified collinear IC AMOD structure [13]. In addition, a small perpendicular component is identified, resulting in an overall elongatedelliptical cycloid $\left(\left|\mathbf{S}_{0}^{\mathrm{Re}}\right| /\left|\mathbf{S}_{0}^{\mathrm{Im}}\right| \sim 0.37\right)$, with its normal canted $\sim 15^{\circ}$ away from the $c$ axis. The new data thus reveal a magnetic structure that combines AMOD and helical properties. The quality of the new refinement reflects in the reduced $\chi^{2}=9.6$ of the polarization matrices being notably decreased with respect to its value in the collinear AMOD (14.7) and the circular helical structures (14.9) [18]. A small misfit [Figs. 2(a) and 2(b)] inevitably originates from a weak nuclear-magnetic-interference term [18].

At $T \rightarrow 0$, the ordered component of the magnetic moments in the elliptical IC AMOD structure is expected to grow at the expense of the disordered one. This can reflect either as a change of the magnetic-reflection intensities, if circular helix is formed, or, alternatively, as additional magnetic reflections with propagation vectors $3 \mathbf{q}$, $5 \mathbf{q}, \ldots$ and intensities $1 / 9,1 / 25, \ldots$ of the first-order magnetic reflections in the case of "squaring" [14]. To detect such changes, single crystal neutron diffraction was performed at $60(3) \mathrm{mK}\left(T / T_{N} \sim 1 / 175\right)$, where we recorded broad $k$ scans of several $h k l \pm \mathbf{q}$ pairs, i.e., intersecting the positions of $h k l \pm n \mathbf{q}, n=3,5, \ldots$, reflections. Surprisingly, we find no significant difference between $60 \mathrm{mK}$ and $2 \mathrm{~K}$ data as well as no trace of higher harmonics [Fig. 2(c)], which implies that both the AMOD ordered component and its disordered counterpart are still present at the lowest accessible temperatures.

Since the neutron diffraction experiments probe only static magnetism, we employed a local-probe $\mu$ SR technique, which is extremely sensitive to internal magnetic fields and can distinguish between fluctuating and static

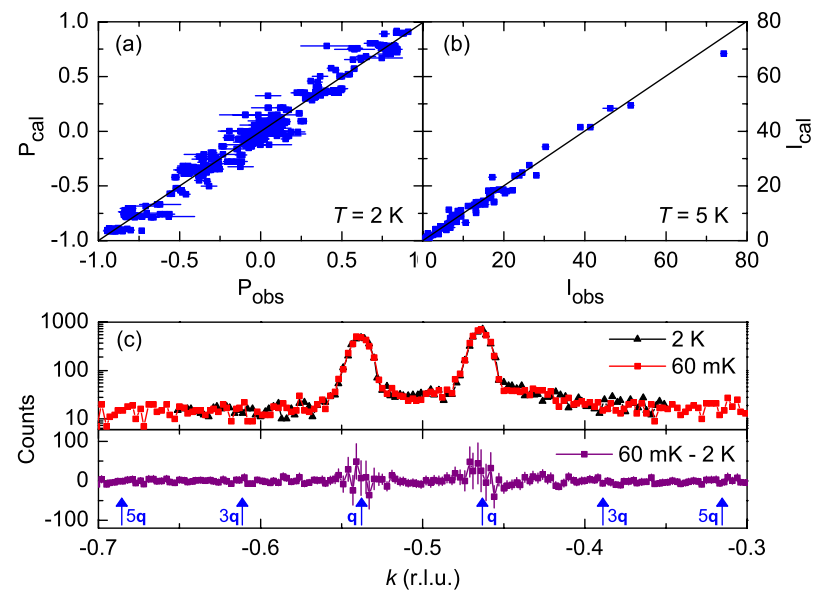

FIG. 2 (color online). Refinement quality for (a) polarization matrices and (b) integrated intensities. (c) Top: $k$ scans at $60(3) \mathrm{mK}$ and $2 \mathrm{~K}$ across $(3.5-0.5 \pm \delta 0)$ reflections, witnessing the absence of higher harmonics, expected to occur at marked positions. Bottom: The difference between the two scans which match within the error bar. 
magnetism, as well as between LRO and static magnetic disorder [20]. The $\mu$ SR experiments were performed on the MUSR instrument at the ISIS facility, Rutherford Appleton Laboratory, United Kingdom, on the same high-purity powder samples as used in our earlier study $[18,21]$. All the data in the following are shown with properly subtracted background signal $(\sim 15 \%)$ [18]. Preliminary results indicated that muons stop at several inequivalent positions [21]. However, to test the magnetic structure model, these must be precisely determined. New measurements were therefore first performed in the paramagnetic state, at $50 \mathrm{~K}$ [Fig. 3(a)], where weak static nuclear magnetic fields are expected to govern the $\mu^{+}$ spin relaxation [20]. Since these fields can be exactly calculated from the crystal structure, they are essential for identification of the muon stopping sites, as demonstrated below. In the case of a single muon stopping site, in a paramagnetic powder sample the muon polarization in zero applied magnetic field $(\mathrm{ZF})$ is given by the Gaussian Kubo-Toyabe relaxation function $G_{\mathrm{KT}}(t, \Delta)=$ $\frac{1}{3}+\frac{2}{3}\left[1-(\Delta t)^{2}\right] \exp \left[-(\Delta t)^{2} / 2\right]$ multiplied by the exponential function $\exp \left[-\lambda_{L} t\right]$. The former accounts for a static Gaussian nuclear field distribution with the width $\Delta / \gamma_{\mu}\left(\gamma_{\mu}=2 \pi \times 135.5 \mathrm{MHz} / \mathrm{T}\right)$, whereas the latter describes a weak dynamical relaxation due to fast electronic fluctuations [20]. The resulting function has a single dip, which is removed only by a fast dynamical relaxation, i.e., $\lambda_{L} \gtrsim \Delta$. Our ZF data exhibit a more complex behavior [Fig. 3(a)], as they require the two-component model
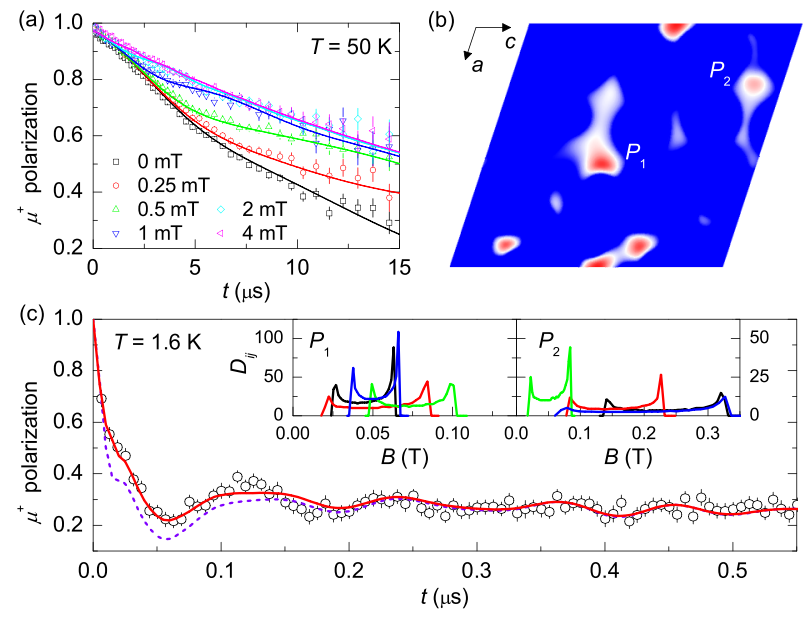

FIG. 3 (color online). (a) Longitudinal-field $\mu \mathrm{SR}$ measurements on powder sample $\mathrm{FeTe}_{2} \mathrm{O}_{5} \mathrm{Br}$ at $50 \mathrm{~K}$ (symbols) and corresponding fits (lines) - see the text. (b) Electrostatic potential at $b=0.15$, i.e., intersecting the most pronounced minima, including $P_{1}$ and $P_{2}$. (c) Zero-field $\mu$ SR measurement at $1.6 \mathrm{~K}$ with corresponding two- (dashed line) and three-component (solid line) fits given by the magnetic structure (see the text). Insets: Calculated field distributions $D_{i j}(B)$ at $P_{i}(i=1,2)$ sites for the four magnetically inequivalent positions $j$ within the unit cell.

$$
G(t)=\left[G_{\mathrm{KT}}\left(t, \Delta_{A}\right)+G_{\mathrm{KT}}\left(t, \Delta_{B}\right)\right] \exp \left[-\lambda_{L} t\right]
$$

This model is supported by measurements in longitudinal applied magnetic fields, where decoupling of the muon relaxation from nuclear-magnetic fields occurs in two steps at $\sim 0.5$ and $\sim 2 \mathrm{mT}$ [Fig. 3(a)], respectively. Simultaneous fit of all $50 \mathrm{~K} \mu \mathrm{SR}$ data to the two-component model, extended for the case of longitudinal applied magnetic fields [20] [Fig. 3(a)], yields the relative occupancies of the two muon stopping sites of 83(3)\% and 17(3)\% with corresponding $\Delta_{A} / \gamma_{\mu}=0.064(5) \mathrm{mT}$ and $\Delta_{B} / \gamma_{\mu}=$ 0.304(5) $\mathrm{mT}$, whereas $\lambda_{L}=0.039(3) \mu \mathrm{s}^{-1}$.

Since $\mu^{+}$are positively charged particles and are generally expected to stop at the electrostatic-potential minima, we calculated the electrostatic potential in the $\mathrm{FeTe}_{2} \mathrm{O}_{5} \mathrm{Br}$ unit cell by using density functional theory [18]. This way several possible stopping sites were identified [Fig. 3(b)], for which dipolar nuclear-magnetic field distributions were calculated [18]. The dominant $\Delta_{A} / \gamma_{\mu}$ is found to be in excellent agreement with nuclear field distributions calculated at two local electrostatic-potential minima $P_{1}=(0.59,0.12,0.43)$ and $P_{2}=(0.25,0.15$, $0.90)$ [Fig. 3(b)]. These are thus assigned as the prime muon stopping sites. On the contrary, $\Delta_{B} / \gamma_{\mu}$ does not agree with calculated distribution at any electrostaticpotential minima [18], so the third (least occupied) muon stopping site remains unassigned.

Identification of the $P_{1}$ and $P_{2}$ stopping sites allows us to calculate local dipolar magnetic fields from the ordered $\mathrm{Fe}$ moments and thus to double check the magnetic structure determined by neutrons. We computed [18] normalized field distributions $D_{i j}(B)$ at four inequivalent sites $(j=$ 1-4) [Wyckoff position 4(e)] for each $P_{i}(i=1,2)$ felt by muons stopping in random unit cells [insets in Fig. 3(c)]. In Fig. 3(c), the ZF $\mu$ SR data collected at $1.6 \mathrm{~K}$ at PSI [21] are shown together with the fit (dashed line) to the corresponding model

$$
\begin{aligned}
G(t)= & \frac{1}{3} \exp \left[-\left(\lambda_{L} t\right)^{\alpha}\right] \\
& +\frac{2}{3} \sum_{i, j}\left[A_{i} \exp \left(-\lambda_{i} t\right) \int_{0}^{\infty} D_{i j}(B) \cos \left(\gamma_{\mu} B t\right) d B\right] .
\end{aligned}
$$

In this expression the first term, commonly called the " $1 / 3$ tail" [20], describes muons in a powder sample, whose initial polarization is parallel to the internal magnetic field and therefore changes only due to fluctuations of this field. This term (dynamical relaxation rate $\lambda_{L}$ and stretch exponent $\alpha$ ) is determined from long-time decay measurements presented in the next paragraph. Now we focus on the second term, which depicts oscillations due to internal fields induced by the LRO magnetic order. These oscillations are damped $\left(\lambda_{i}\right)$ by spin fluctuations and/or static relaxation resulting from a distribution of muon stopping 
positions around $P_{i}$ [18], with occupancy $A_{i}\left(\sum_{i} A_{i}=1\right)$. The fit yields $\lambda_{1}=0.60(5) \mu \mathrm{s}^{-1}, \lambda_{2}=50(8) \mu \mathrm{s}^{-1}$, and $A_{2} / A_{1}=1.4(1)$. Most importantly, our model excellently accounts for the experimental oscillation frequencies of the muon polarization determined by $D_{i j}(B)$ (no adjustable parameters) and thus firmly affirms the elongated-elliptical IC AMOD state. The small discrepancy [Fig. 3(c)] is most likely due to the neglected $17(3) \%$ of muons, with unknown stopping position $\left(\Delta_{B}\right)$. Indeed, adding a third component with $A_{3}=17 \%$ and its decay approximated by $\exp \left(-\lambda_{3} t\right)$ leads to a perfect agreement with the experiment [Fig. 3(c)]. The improved model yields $\lambda_{3}=$ 11(1) $\mu \mathrm{s}^{-1}$, while parameters $\lambda_{1}, \lambda_{2}$, and $A_{2} / A_{1}$ stay within the error bars of the two-component model. The agreement of the neutron and the $\mu$ SR results proves that $\mu \mathrm{SR}$ probes the intrinsic magnetic properties and can thus provide an invaluable insight into spin dynamics of the $\mathrm{FeTe}_{2} \mathrm{O}_{5} \mathrm{Br}$ system.

In the case of completely static $\left(\lambda_{L}=0\right)$ local magnetic fields, the $1 / 3$ tail persists in the $t \rightarrow \infty$ limit [Eq. (3)]. Since preliminary ZF $\mu$ SR data implied its decay [21], we extended these measurements to longer times and very low temperatures [Fig. 4(a)]. The new data confirm the decay of the $1 / 3$ tail and clearly show its persistence down to the lowest accessible temperature of 53(3) $\mathrm{mK}$, i.e., $T / T_{N} \sim$ $1 / 200$. Since this decay can only be of dynamical origin, it unambiguously proves that muons, experiencing static magnetic fields due to the IC AMOD order, also experience local-field fluctuations, i.e., revealing the coexistence of PSD and LRO at $T \rightarrow 0$.
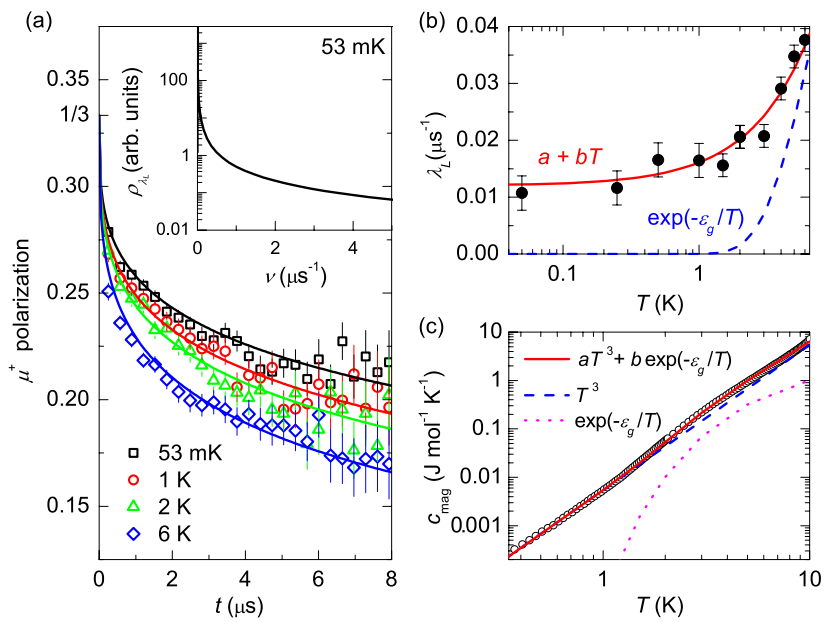

FIG. 4 (color online). (a) Muon depolarization (symbols) at low temperatures, indicating the presence of PSD, and corresponding fits (lines) to the stretched-exponential function $\exp \left[-\left(\lambda_{L} t\right)^{0.3}\right]$. Inset: Relaxation rate distribution at $53 \mathrm{mK}$ determined from fit (see the text). (b) Extracted relaxation rates, showing linear temperature dependence (solid line). For comparison, activation behavior (dashed line) for gapped $\left(\epsilon_{g}=\right.$ $11.5 \mathrm{~K}$ ) magnon excitations is shown. (c) Temperature dependence of the magnetic contribution to the specific heat, $c_{\text {mag }}(T)$, together with corresponding fits (see the text for details).
To obtain deeper insight into PSD, we focus on the dynamic part of the $\mu$ SR signal, i.e., muon polarization for $t>0.5 \mu \mathrm{s}$, where the second term in Eq. (3) is already relaxed [Fig. 3(b)]. These data are fitted with $G(t)=$ $\frac{1}{3} \exp \left[-\left(\lambda_{L} t\right)^{\alpha}\right]$ [Fig. 4(a)], where $\alpha$ accounts for the dynamical relaxation rate distribution $\rho_{\lambda_{L}}(\nu)$, related to the stretched-exponential function by the Laplace transform $\exp \left[-\left(\lambda_{L} t\right)^{\alpha}\right]=\int_{0}^{\infty} \rho_{\lambda_{L}}(\nu) \exp [-(\nu t)] d \nu$ [22]. A small $\alpha=0.30$ (2), found temperature independent below $T_{N}$, indicates a very broad $\rho_{\lambda_{L}}(\nu)$ [inset in Fig. 4(a)], which most likely reflects a distribution and dynamical nature of the disordered parts of the magnetic moments in the AMOD state. Additionally, $\rho_{\lambda_{L}}(\nu)$ can be broadened, because three different muon stopping sites are present. The obtained $\lambda_{L}$ [Fig. 4(b)] shows a linear temperature dependence and, most importantly, converges to a finite zero-temperature value $\lambda_{L}^{0}=0.012(2) \mu \mathrm{s}^{-1}$, characteristic of PSD. We note that, in spite of the small $\lambda_{L}^{0}$, the broad $\rho_{\lambda_{L}}(\nu)$ spans far into the $\mu \mathrm{SR}$ time window $(\nu>$ $\left.0.1 \mu \mathrm{s}^{-1}\right)$, as evident from substantial experimental decay of the $1 / 3$ tail at early times [Fig. 4(a)].

In LRO antiferromagnets, $\lambda_{L}(T)$ is expected to follow either $T^{n}$ dependence with $n>2$ for $T \gg \epsilon_{g}$ or $\exp \left(-\epsilon_{g} / T\right)$ dependence for $T \ll \epsilon_{g}$ [11,23-25]. Here $\epsilon_{g}$ is the magnon energy gap. Considering $\epsilon_{g}=11.5 \mathrm{~K}$, as determined in the recent antiferromagnetic-resonance study [12], $\lambda_{L}(T)$ should change exponentially with temperature in the inspected temperature range, which is clearly not the case [Fig. 4(b)]. Hence, $\lambda_{L}(T)$ signifies magnetic excitations, which are different from the usual magnon modes in LRO states. Finite relaxation at $T \rightarrow 0$ is typically found in gapless spin liquids where it is ascribed to quantum spin fluctuations $[2,3,26,27]$. In $\mathrm{FeTe}_{2} \mathrm{O}_{5} \mathrm{Br}$, these most probably originate from the disordered component of the magnetic moment at each Fe site, which naturally accompanies the ordered component in an IC AMOD structure. The dual nature of the ground state is confirmed by the magnetic contribution $c_{\text {mag }}(T)$ to specific heat [16], which is proportional to $T^{3}$ below $T_{N}$ and exhibits an additional broad hump around $3 \mathrm{~K}$ [Fig. 4(c)]. The former implies three-dimensional antiferromagnetic gapless excitations [5,28-30], while the latter reveals an additional thermally activated $\exp \left(-\epsilon_{g} / T\right)$ term due to the gapped magnetic excitations [29].

Finally, we point out similar spin dynamics in the magnetic ground state of volborthite [25], where spin-densitywave-like modulation [25] and IC spin correlations [31] were found. Moreover, recent calculations [32] showed that volborthite, like $\mathrm{FeTe}_{2} \mathrm{O}_{5} \mathrm{Br}$ [12], has to be treated as a frustrated coupled-spin-chain system. This suggests that such systems are keen to form an IC AMOD magnetic ground state, which offers a sound phenomenological explanation of the coexistence of PSD and LRO.

In conclusion, we have found that in $\mathrm{FeTe}_{2} \mathrm{O}_{5} \mathrm{Br}$ the elongated-elliptical IC AMOD magnetic structure persists 
down to $T / T_{N} \sim 1 / 200$, as a result of frustrated chain topology. In this LRO state, fluctuations of the remaining disordered spin component at each magnetic site are intrinsic, and they manifest as PSD at $T \rightarrow 0$. Similar observations in volborthite suggest that IC AMOD magnetic order is a natural habitat for PSD and can serve as a model structure inherently encompassing the intriguing coexistence of PSD and LRO. This conjecture could be tested on linarite, for which frustrated chains were recently reported to induce a LRO AMOD structure [33]. Further in-depth theoretical investigations are required to account for PSD in IC AMOD structures on the microscopic level.

We thank M. J. P. Gingras for valuable comments. This research project has been partially supported by the Slovenian Research Agency Project No. J1-2118, by the Swiss National Science Foundation Project No. 200021129899, and by the European Commission under the 7th Framework Program through the "Research Infrastructures" action of the "Capacities" Program, NMI3-II Grant No. 283883. Contract No. CPCSA_INFRA-2008-1.1.1 No. 226507-NMI3.

[1] Introduction to Frustrated Magnetism, edited by C. Lacroix, P. Mendels, and F. Mila (Springer-Verlag, Berlin, 2011).

[2] Y. J. Uemura et al., Phys. Rev. Lett. 73, 3306 (1994).

[3] L. Balents, Nature (London) 464, 199 (2010).

[4] A. Yaouanc, P. Dalmas de Réotier, V. Glazkov, C. Marin, P. Bonville, J. A. Hodges, P. C. M. Gubbens, S. Sakarya, and C. Baines, Phys. Rev. Lett. 95, 047203 (2005).

[5] J. Lago, T. Lancaster, S. J. Blundell, S. T. Bramwell, F. L. Pratt, M. Shirai, and C. Baines, J. Phys. Condens. Matter 17, 979 (2005).

[6] X. Zheng, H. Kubozono, K. Nishiyama, W. Higemoto, T. Kawae, A. Koda, and C. N. Xu, Phys. Rev. Lett. 95, 057201 (2005).

[7] P. Dalmas de Réotier et al., Phys. Rev. Lett. 96, 127202 (2006).

[8] S. R. Dunsiger, R. F. Kiefl, J. A. Chakhalian, J. E. Greedan, W. A. MacFarlane, R. I. Miller, G. D. Morris, A. N. Price, N. P. Raju, and J. E. Sonier, Phys. Rev. B 73, 172418 (2006).

[9] S. R. Giblin, J. D. M. Champion, H. D. Zhou, C. R. Wiebe, J.S. Gardner, I. Terry, S. Calder, T. Fennell, and S.T. Bramwell, Phys. Rev. Lett. 101, 237201 (2008).

[10] K. C. Rule, G. Ehlers, J. S. Gardner, Y. Qiu, E. Moskvin, K. Kiefer, and S. Gerischer, J. Phys. Condens. Matter 21, 486005 (2009).
[11] P. A. McClarty, J. N. Cosman, A. G. Del Maestro, and M. J.P. Gingras, J. Phys. Condens. Matter 23, 164216 (2011).

[12] M. Pregelj et al., Phys. Rev. B 86, 054402 (2012).

[13] M. Pregelj, O. Zaharko, A. Zorko, Z. Kutnjak, P. Jeglič, P. J. Brown, M. Jagodič, Z. Jagličić, H. Berger, and D. Arčon, Phys. Rev. Lett. 103, 147202 (2009).

[14] S.-M. Choi, J. W. Lynn, D. Lopez, P. L. Gammel, P. C. Canfield, and S. L. Bud'ko, Phys. Rev. Lett. 87, 107001 (2001).

[15] G. Lawes et al., Phys. Rev. Lett. 93, 247201 (2004).

[16] M. Pregelj et al., Phys. Rev. B 82, 144438 (2010).

[17] Neutron Scattering from Magnetic Materials, edited by T. Chatterji (Elsevier, Amsterdam, 2006).

[18] See Supplemental Material at http://link.aps.org/ supplemental/10.1103/PhysRevLett.109.227202 for details of neutron scattering experiments and magnetic structure refinement, background determination of the $\mu \mathrm{SR}$ data, nuclear- and electronic-magnetic-field calculations, density-functional-theory calculations, and modeling of the crystal-lattice contribution to the specific heat.

[19] O. Zaharko, M. Pregelj, D. Arčon, P. J. Brown, D. Chernyshov, U. Stuhr, and H. Berger, J. Phys. Conf. Ser. 211, 012002 (2010).

[20] A. Youanc and P. Dalmas de Réotier, Muon Spin Rotation, Relaxation and Resonance (Oxford University, New York, 2011).

[21] A. Zorko, M. Pregelj, H. Berger, and D. Arčon, J. Appl. Phys. 107, 09D906 (2010).

[22] D. C. Johnston, Phys. Rev. B 74, 184430 (2006).

[23] T. Moriya, Prog. Theor. Phys. 16, 23 (1956).

[24] F. Mila and T. M. Rice, Phys. Rev. B 40, 11382 (1989).

[25] M. Yoshida, M. Takigawa, H. Yoshida, Y. Okamoto, and Z. Hiroi, Phys. Rev. Lett. 103, 077207 (2009).

[26] J. S. Gardner et al., Phys. Rev. Lett. 82, 1012 (1999).

[27] A. Keren, Y. J. Uemura, G. Luke, P. Mendels, M. Mekata, and T. Asano, Phys. Rev. Lett. 84, 3450 (2000).

[28] L. J. De Jongh and A. R. Miedema, Adv. Phys. 50, 947 (2001).

[29] J. A. Quilliam, K. A. Ross, A. G. Del Maestro, M. J.P. Gingras, L. R. Corruccini, and J. B. Kycia, Phys. Rev. Lett. 99, 097201 (2007).

[30] S. Ohkoshi, H. Tokoro, and K. Hashimoto, Coord. Chem. Rev. 249, 1830 (2005).

[31] G. J. Nilsen, F. C. Coomer, M. A. de Vries, J. R. Stewart, P. P. Deen, A. Harrison, and H. M. Rønnow, Phys. Rev. B 84, 172401 (2011).

[32] O. Janson, J. Richter, P. Sindzingre, and H. Rosner, Phys. Rev. B 82, 104434 (2010).

[33] B. Willenberg et al., Phys. Rev. Lett. 108, 117202 (2012). 